Hope College

Hope College Digital Commons

Faculty Publications

7-10-2012

\title{
The Complexity of Pebbling in Diameter Two Graphs*
}

Charles A. Cusack

HopeCollege, cusack@hope.edu

Timothy Lewis

Hope College

Daniel Simpson

Hope College

Samuel Taggart

Oberlin College

Follow this and additional works at: https://digitalcommons.hope.edu/faculty_publications

Part of the Computer Sciences Commons

\section{Recommended Citation}

Cusack, Charles A., Timothy Lewis, Daniel Simpson and Samuel Taggart. "The Complexity of Pebbling in Diameter Two Graphs*." SIAM Journal on Discrete Mathematics 26, no. 3 (2012): 919-928.

This Article is brought to you for free and open access by Hope College Digital Commons. It has been accepted for inclusion in Faculty Publications by an authorized administrator of Hope College Digital Commons. For more information, please contact digitalcommons@hope.edu. 


\title{
THE COMPLEXITY OF PEBBLING IN DIAMETER TWO GRAPHS*
}

\author{
CHARLES A. CUSACK ${ }^{\dagger}$, TIMOTHY LEWIS ${ }^{\dagger}$, DANIEL SIMPSON ${ }^{\dagger}$, AND SAMUEL
} TAGGART T $^{\ddagger}$

\begin{abstract}
Given a simple, connected graph, a pebbling configuration is a function from its vertex set to the nonnegative integers. A pebbling move between adjacent vertices removes two pebbles from one vertex and adds one pebble to the other. A vertex $r$ is said to be reachable from a configuration if there exists a sequence of pebbling moves that places one pebble on $r$. A configuration is solvable if every vertex is reachable. We prove tight bounds on the number of vertices with two and three pebbles that an unsolvable configuration on a diameter two graph can have in terms of the size of the graph. We also prove that determining reachability of a vertex is NP-complete, even in graphs of diameter two.
\end{abstract}

Key words. graph pebbling, diameter two, NP-complete

AMS subject classifications. 68Q17, 68R05, 68R10

DOI. $10.1137 / 11084412 \mathrm{X}$

1. Introduction. Let $G=(V, E)$ be a simple, connected graph with vertex set $V$, edge set $E$, and $n(G)=|V|$ (or just $n$, if the graph is clear from context). The distance between $u, v \in V$ (written $\operatorname{dist}(u, v)$ ) is the number of edges in the shortest path between $u$ and $v$. The diameter of a graph is the maximum distance between any two vertices in $G$. If $U \subseteq V$ and $v \in V$, we define $N_{U}(v)=\{u \in U \mid u$ is adjacent to $v\}$. That is, $N_{U}(v)$ are the neighbors of $v$ from $U$.

A pebbling configuration (or just configuration) is a function $C: V \rightarrow \mathbb{N}$ (the nonnegative integers), where $C(v)$ represents the number of "pebbles" placed on vertex $v$. For $k \in \mathbb{N}$ we define $V_{k}=\{v \in V \mid C(v)=k\}$. That is, $V_{k}$ is the set of vertices that have $k$ pebbles. Given $u, v \in V$, with $u$ and $v$ adjacent, we define a pebbling move from $u$ to $v$ (denoted $(u, v)$ ) to be the process of removing two pebbles from $u$ and adding one pebble to $v$. If $\sigma$ is a sequence of pebbling moves, then $C_{\sigma}$ is the configuration after the pebbling moves from $\sigma$ have been executed starting with configuration $C$. A sequence of pebbling moves is executable if after each successive move every vertex has a nonnegative number of pebbles on it. From now on we will assume that a sequence of pebbling moves is executable unless otherwise stated.

We will employ several more definitions which will help us rigorously characterize sequences of pebbling moves. A sequence $\sigma$ that accomplishes some goal (e.g., places one pebble on a specified vertex) is minimal if removing any pebbling moves from $\sigma$ renders the goal unattainable. Likewise, $\sigma$ is a minimum sequence of pebbling moves if it accomplishes the goal in the smallest possible number of pebbling moves. The following lemma is a restatement of Lemma 2.7 in [10].

Lemma 1. Let $C$ be a pebbling configuration and $\sigma$ a minimal pebbling sequence that places $k>C(r)$ pebbles on some vertex $r$. If the moves from $\sigma$ are viewed as directed edges on a graph, $\sigma$ is acyclic.

*Received by the editors August 11, 2011; accepted for publication (in revised form) April 6, 2012; published electronically July 10, 2012. This work was supported by the NSF under grant DUE0851293.

http://www.siam.org/journals/sidma/26-3/84412.html

${ }^{\dagger}$ Department of Computer Science, Hope College, Holland, MI 49422 (cusack@hope.edu, timothy. lewis@hope.edu, daniel.simpson@hope.edu).

${ }_{\ddagger}^{\ddagger}$ Department of Mathematics, Oberlin College, Oberlin, OH 44074 (Samuel.Taggart@oberlin.edu). 
Given a pebbling configuration $C$, we say $r \in V$ is reachable if there exists a pebbling sequence $\sigma$ such that $C_{\sigma}(r)>0$. A configuration is solvable if every vertex is reachable. For example, a diameter two graph with four pebbles on any vertex is guaranteed to be solvable. The computational problems of determining reachability (which we will call REACHABle) and solvability (SOLVABLE) have come to the attention of researchers in the last decade. It is not difficult to see that these problems are computationally equivalent. The fact that REACHABLE is NP-complete has been proven at least three times, starting with Hurlbert and Kierstead [9]. Subsequent to this Watson [11] and (independently) Milans and Clark [10] proved that REACHABLE and several other graph pebbling problems are NPcomplete. We will prove that REACHABLE is NP-complete once more with a reduction inspired by that of Milans and Clark, but which is simpler and smaller. Our reduction allows for an additional reduction to reachability in diameter two graphs (D2-REACHABLE).

The pebbling number, denoted $\pi(G)$, is the smallest number of pebbles such that if there are $\pi(G)$ pebbles on the graph, the graph is solvable no matter how the pebbles are placed. Determining pebbling number (PebBling-Number) was shown in [10] to be $\Pi_{2}^{P}$-complete.

Graph pebbling was first suggested by Lagarias and Saks and developed by Chung in [4], where the pebbling number was used as a tool for studying the zero-sum sequence problem. Since then, it has received attention from researchers interested in its properties outside of a number-theoretic setting. In her paper, Chung sets forth one particular conjecture, dubbed Graham's conjecture, that has captured the attention of numerous researchers. The conjecture states that for any two graphs $G_{1}$ and $G_{2}$, $\pi\left(G_{1} \square G_{2}\right) \leq \pi\left(G_{1}\right) \pi\left(G_{2}\right)$, where $\square$ denotes the Cartesian product. Though unproven in general, it has been verified for various families of graphs. For a more complete survey of results on this problem and other generalizations of graph pebbling, [7] and [8] are excellent resources.

Because many pebbling problems are computationally hard, a few researchers have turned their attention to the special case of diameter two graphs. In a probabilistic sense, almost every graph has diameter two [5], so results with this restriction still shed light on pebbling in graphs as a whole. Restricting a graph's diameter causes the pebbling number to be much better behaved, with all diameter two graphs having pebbling number $\pi(G)=n$ (called Class 0 ) or $\pi(G)=n+1$ (Class 1 ).

Clarke, Hochberg, and Hurlbert give a characterization of Class 1 graphs in [5], which Blasiak and Schmitt correct in [3]. While the characterization discussed in these papers implies that the pebbling number for diameter two graphs can be computed in polynomial time, [2] provides an explicit algorithm, verifying that the problem is in P. Herscovici, Hester, and Hurlbert give a slightly improved algorithm in [6]. A polynomial-time algorithm to solve D2-REACHABLE on all Class 1 graphs and Class 0 graphs with small connectivity is given in [2]. These results suggested that a polynomial-time algorithm for D2-REACHABLE would be possible. We show here that this is in fact not possible unless $\mathrm{P}=\mathrm{NP}$, by proving that D2-REACHABLE is NP-complete.

On the way to our main result we prove tight bounds on $\left|V_{2}\right|$ and $\left|V_{3}\right|$ for unsolvable configurations on diameter two graphs. These results have at least two implications. First, the worst case complexity of the algorithm in [2] is improved, although the worst case is still exponential. Second, they may lead to an improvement of the threshold for diameter two graphs. Given a sequence of graphs $\mathcal{G}=\left\{G_{1}, G_{2}, \ldots, G_{n}, \ldots\right\}$, where $G_{n}$ has $n$ vertices, a threshold for $\mathcal{G}$ is a function $t(n)$ such that if asymptotically more 
(less) than $t(n)$ pebbles are placed randomly on $G_{n}$, then the probability that the configuration is solvable (unsolvable) approaches 1 as $n$ approaches infinity. For more on thresholds, see [1].

2. Constructions. In this section we describe two constructions that will be used to prove a few bounds and show that D2-REACHABLE is NP-complete.

2.1. An important diameter two graph. For $k \geq 2$, define $H(k)$ to be the graph whose vertices are the subsets of $\{1, \ldots, k\}$ with one or two elements and whose edges consist of pairs of vertices whose sets intersect. Label each vertex $v_{A}$, where $A$ is the subset corresponding to the vertex. If $A=\{a, b\}$, then we write $v_{A}$ as $v_{a, b}$, and similarly if $A=\{a\}$, we will write $v_{a}$. We can describe the edges in $H(k)$ using this new notation: given sets $A$ and $B,\left\{v_{A}, v_{B}\right\} \in E(H(k))$ if and only if $A \cap B \neq \emptyset$. Notice that $n(H(k))=k+\left(\begin{array}{c}k \\ 2\end{array}\right)=\left(\begin{array}{c}k+1 \\ 2\end{array}\right)$.

Lemma 2. $H(k)$ has diameter two.

Proof. Let $v_{A}, v_{B} \in V(H(k))$, with $v_{A} \neq v_{B}$. If $A \cap B \neq \emptyset$, then $v_{A}$ is adjacent to $v_{B}$. If $A \cap B=\emptyset$, then let $S=\{a, b\}$, where $a \in A$ and $b \in B$. Note that $S \cap A \neq \emptyset$ and $S \cap B \neq \emptyset$. Therefore $v_{A}$ and $v_{B}$ are adjacent to $v_{S}$. In either case the maximum distance between $v_{A}$ and $v_{B}$ is 2 .

Lemma 3. Let $C$ be a configuration of pebbles on $H(k)$ such that $C\left(v_{a}\right) \leq 3$ and $C\left(v_{a, b}\right)=0$ for all $a, b \in\{1, \ldots, k\}$ with $a \neq b$. Then for any pebbling sequence $\sigma$, $C_{\sigma}\left(v_{a}\right) \leq C\left(v_{a}\right)$ for all $a \in\{1, \ldots, k\}$.

Proof. Let $\sigma$ be a minimum sequence of pebbling moves that adds one pebble to $v_{a}$ for some $a$. Without loss of generality, assume $a=1$ and that the last move is $\left(v_{1,2}, v_{1}\right)$. Clearly $\sigma$ does not add any pebbles to $v_{b}$ for any $b \neq 1$, since this would imply a shorter sequence which adds a pebble to a vertex $v_{b}$, contradicting the minimality of $\sigma$. Two pebbles must be placed on $v_{1,2}$, at most one of which can be from $v_{2}$ since $C\left(v_{2}\right) \leq 3$. Thus one pebble must come from $v_{1, b}$ or $v_{2, b}$ for some $b$. If the pebble came from $v_{1, b}$, then moving from $v_{1, b}$ to $v_{1}$ directly yields a shorter sequence. Similarly, if one came from $v_{2, b}$, then moving to $v_{2}$ yields a shorter sequence.

2.2. Embedding a graph in $\boldsymbol{H}(\boldsymbol{k})$. Let $G=(V, E)$ be a graph with $V=$ $\left\{v_{1}, \ldots, v_{n}\right\}$ such that $n \geq 2$. Define $\mathcal{H}(G)$ to be the graph with vertex set $V \cup V^{\prime}$, where $V^{\prime}=\left\{v_{a, b}: v_{a}, v_{b} \in V\right.$ and $\left.v_{a} \neq v_{b}\right\}$. The edge set of $\mathcal{H}(G)$ will be $E \cup E^{\prime}$, where $E^{\prime}=E(H(n))$. Given a configuration $C$ on $G$, we can define configuration $C^{\prime}$ on $\mathcal{H}(G)$ by defining $C^{\prime}(v)=C(v)$ if $v \in V$ and 0 otherwise. Essentially, $\mathcal{H}(G)$ embeds $G$ into the 1-element subsets of $H(n)$.

Lemma 4. $\mathcal{H}(G)$ has diameter at most 2.

Proof. Note that $H(n)$ is a subgraph of $\mathcal{H}(G)$ with the same vertex set. Because the addition of edges cannot increase a graph's diameter, the diameter of $\mathcal{H}(G)$ is no greater than the diameter of $H(n)$. Thus, by Lemma $2, \mathcal{H}(G)$ has at most diameter two.

Lemma 5. Let $C$ be a configuration on $G$ such that for any pebbling sequence $\sigma$, $C_{\sigma}(v) \leq 3$ for all $v \in V$. Then any vertex $r \in V$ is reachable in $G$ from $C$ if and only if $r$ is reachable in $\mathcal{H}(G)$ from $C^{\prime}$.

Proof. If $r$ is reachable in $G$ from $C$, then we can use the same sequence of moves to reach $r$ in $\mathcal{H}(G)$ from $C^{\prime}$.

Conversely, let $\sigma$ be a minimal sequence of pebbling moves on $\mathcal{H}(G)$ that places one pebble on $r \in V$. Order $\sigma$ such that before the first move along an edge in $E^{\prime}$ occurs, no more moves along edges in $E$ are possible (that is, without first making one or more moves in $\left.E^{\prime}\right)$. Let $\psi$ be the subsequence containing these moves in $E$, 
and $\rho$ the next subsequence of moves in $E^{\prime}$. Because $C_{\psi}^{\prime}(v) \leq 3$ when $v \in V$ and $C_{\psi}^{\prime}(u)=0$ when $u \in V^{\prime}$, Lemma 3 implies that $\rho$ does not add any pebbles to any $v \in V$, including $r$. This implies that no additional moves in $E$ are possible after $\psi$ and $\rho$, so these are all of the moves from $\sigma$. But the moves from $\rho$ do not place a pebble on $r$, so $\sigma$ being minimal implies that $\rho$ is empty. Thus $r$ is reachable in the induced subgraph of $\mathcal{H}(G)$ with the vertex set $V$, which is the graph $G$.

3. Bounds. In this section we will develop tight bounds on $\left|V_{2}\right|$ and $\left|V_{3}\right|$ for unsolvable configurations on diameter two graphs. For each of these we will utilize the constructions just developed to create unsolvable configurations on diameter two graphs that meet the bound. We will then show that these graphs are the smallest possible.

3.1. Vertices with three pebbles. This case is fairly straightforward.

THEOREM 6 . The smallest unsolvable diameter two graph that contains $k$ vertices with three pebbles has $\left(\begin{array}{c}k+2 \\ 2\end{array}\right)$ vertices.

Proof. Let $C$ be a pebbling configuration on $H(k+1)$ such that $C\left(v_{i}\right)=3$ for all $i \in\{1, \ldots, k\}$ and $C(v)=0$ otherwise. $H(k+1)$ has $\left(\begin{array}{c}k+2 \\ 2\end{array}\right)$ vertices, and by Lemma 3 $v_{k+1}$ is unreachable from $C$.

Let $G$ be a diameter two graph with an unsolvable pebbling configuration $C$ such that $\left|V_{3}\right|=k \geq 1$. Then there must be at least one vertex $r$ in $G$ that is not reachable. Let $K=V_{3} \cup\{r\}$. If any elements of $K$ are adjacent or if three or more elements of $K$ are adjacent to the same vertex, then $G$ is solvable from $C$. Since $G$ has diameter two, for every pair of vertices $u, v \in K$ there must be a unique vertex that is adjacent to both $u$ and $v$. The number of these pairs is $\left(\begin{array}{c}|K| \\ 2\end{array}\right)$, so $n(G) \geq|K|+\left(\begin{array}{c}|K| \\ 2\end{array}\right)=k+1+\left(\begin{array}{c}k+1 \\ 2\end{array}\right)=\left(\begin{array}{c}k+2 \\ 2\end{array}\right)$.

Solving for $k$ yields the following corollary.

Corollary 7. A configuration $C$ on a diameter two graph $G$ with $n$ vertices is solvable if $\left|V_{3}\right|>\sqrt{2 n+1 / 4}-3 / 2$.

3.2. Vertices with two pebbles. The case for $\left|V_{2}\right|$ is a little more involved and requires modifying our previous construction slightly.

THEOREM 8. The smallest unsolvable diameter two graph that contains $k$ vertices with two pebbles has $\left(\begin{array}{c}k+2 \\ 2\end{array}\right)-\lfloor k / 2\rfloor$ vertices.

We will prove this result by developing a construction that meets the requirements of the theorem and proving that it is the smallest graph that does so.

For $k \geq 1$ define $G=(V, E)$, where $V=\left\{v_{1}, \ldots, v_{k+1}\right\}$ and $E=\left\{v_{i}, v_{i+1}\right\}$ for all $i \equiv 0 \bmod 2$ (i.e., $\left\{v_{2}, v_{3}\right\},\left\{v_{4}, v_{5}\right\}, \ldots$ ). Then define $H_{2}(k)$ to be the induced subgraph of $\mathcal{H}(G)$ with the vertex set $V(\mathcal{H}(G)) \backslash\left\{v_{a, b}:\left\{v_{a}, v_{b}\right\} \in E\right\}$. The number of edges in $G$ is $\lfloor k / 2\rfloor$, so the number of vertices in $H_{2}(k)$ is $\left(\begin{array}{c}k+2 \\ 2\end{array}\right)-\lfloor k / 2\rfloor$.

Lemma 9. $H_{2}(k)$ has diameter two.

Proof. Let $v_{A}, v_{B} \in V$, where $v_{A} \neq v_{B}$. If $A \cap B \neq \emptyset$, then $v_{A}$ is adjacent to $v_{B}$. If $|A|=|B|=1$, let $A=\{a\}$ and $B=\{b\}$. Then either $v_{A}$ and $v_{B}$ are adjacent to each other or both are adjacent to $v_{a, b}$. If, without loss of generality, $|A|=2$, then let $A=\{a, c\}$ and $b \in B$. Then either $v_{a, b} \in V$ or $v_{c, b} \in V$ (or both). Thus $\operatorname{dist}\left(v_{A}, v_{B}\right) \leq 2$.

LEMma 10. Let $C$ be the configuration on $H_{2}(k)$ such that $C\left(v_{i}\right)=2$ for $i>1$ and $C(v)=0$ otherwise. $H_{2}(k)$ is unsolvable from $C$.

Proof. In $G, v_{1}$ is not reachable and every other vertex can accumulate no more than three pebbles. By Lemma $5, v_{1}$ is not reachable in $\mathcal{H}(G)$. Since $H_{2}(k)$ is a subgraph of $\mathcal{H}(G), v_{1}$ is not reachable in $H_{2}(k)$. 


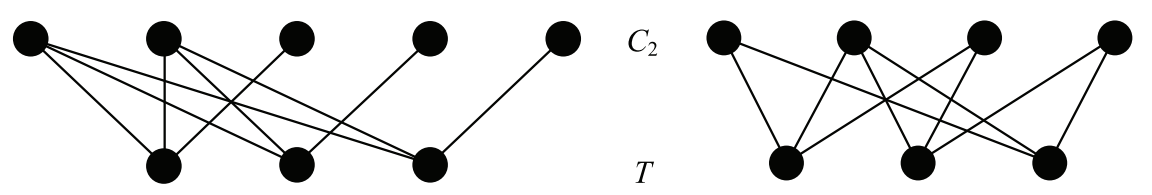

FIG. 1. A triple chain (left) and a minal (right).

For the remainder of this section let $C$ be an unsolvable configuration on a diameter two graph $G$, let some vertex $r$ be unreachable, and let $\left|V_{2}\right|=k \geq 1$. Define an equivalence relation $\mathcal{R}$ on $V-V_{2}-\{r\}$ by $x \sim y$ if $N_{V_{2}}(x)=N_{V_{2}}(y)$. Let $X$ be a set of representatives of the equivalence classes of $\mathcal{R}$, and let $P$ and $T$ be the subsets of $X$ whose vertices are adjacent to two and three elements of $V_{2}$, respectively. Each vertex $x \in P \cup T$ can be uniquely labeled by $N_{V_{2}}(x)$. For example, if $N_{V_{2}}(x)=\{a, b, c\}$, then $x=\{a, b, c\}$.

Lemma 11. If $x, y \in T$, then $|x \cap y| \neq 1$.

Proof. Assume otherwise, with $x=\{a, b, c\}$ and $y=\{c, d, e\}$. The moves $(a, x)$, $(b, x),(x, c),(d, y),(e, y),(y, c)$ place two additional pebbles on $c$. So $c$ now has four pebbles, and $G$ is solvable from $C$, a contradiction.

COROLlary 12. Let $x, y, z \in T$ such that $|x \cap y|=2$. Then either $|x \cap z|=$ $|y \cap z|=2$ or $|x \cap z|=|y \cap z|=0$.

The previous corollary implies that $T$ can be partitioned as $T=\cup_{i=1}^{l} T_{i}$ such that if $x, y \in T_{i}$, then $|x \cap y|=2$, and if $x \in T_{i}$ and $y \in T_{j}$, then $|x \cap y|=0$ when $i \neq j$. More importantly, this partition of $T$ defines a partition of $N_{V_{2}}(T)$ in the obvious way.

Lemma 13. If $a, b \in V_{2}$ are adjacent and $b$ is adjacent to $x \in T$, then $a$ is adjacent to $x$.

Proof. Let $a, b \in V_{2}$ be adjacent, and let $x=\{b, c, d\} \in T$. If $a \notin\{c, d\}$, then the moves $(c, x),(d, x),(x, b),(a, b)$ place two additional pebbles on $b$. So $b$ now has four pebbles, and $G$ is solvable from $C$, a contradiction. Therefore either $a=c$ or $a=d$ and $a$ is adjacent to $x$.

Lemma 14. For every $x \in V_{2} \cup T$ there is a vertex $u_{x} \notin V_{2} \cup P \cup T$ that is adjacent to $x$ and $r$ that is not adjacent to any other vertex in $V_{2} \cup T$.

Proof. Since $G$ has diameter two every $x \in V_{2} \cup T$ must be adjacent to a vertex $u_{x}$ also adjacent to $r$. Since $u_{x}$ is adjacent to $r$, it cannot be in $V_{2} \cup P \cup T$. Assume for some $x, y \in V_{2} \cup T$ that $u_{x}$ is adjacent to both $x$ and $y$.

If $x, y \in V_{2}$, then the moves $\left(x, u_{x}\right),\left(y, u_{x}\right),\left(u_{x}, r\right)$ place a pebble on $r$.

If $x \in V_{2}$ and $y=\{a, b, c\} \in T$, without loss of generality assume $x \notin\{b, c\}$. Then the moves $\left(x, u_{x}\right),(b, y),(c, y),\left(y, u_{x}\right),\left(u_{x}, r\right)$ place a pebble on $r$. A similar argument works if $y \in V_{2}$ and $x \in T$.

If $x=\{a, b, c\}, y=\{d, e, f\} \in T$, then either $x \cap y=\emptyset$ or, without loss of generality, $e=a$ and $f=b$. In either case, the moves $(a, x),(c, x),(f, y),(d, y)$, $\left(x, u_{x}\right),\left(y, u_{x}\right),\left(u_{x}, r\right)$ place a pebble on $r$.

In all cases we get a contradiction, so $u_{x}$ cannot be adjacent to both $x$ and $y$.

Let $R \subseteq T$ be an element of the partition defined after Corollary 12. If every element of $R$ is adjacent to the same $a, b \in V_{2}$, then $R$ is called a triple chain. Otherwise we call $R$ a minal. See Figure 1 . Notice that a minal must contain at least three vertices.

Lemma 15. Let $M$ be a minal and $S \subseteq V_{2}$ be the set of all vertices in $V_{2}$ adjacent to any vertex in $M$. Then $|S|=4$ and $|M| \in\{3,4\}$. 
Proof. Let $\{a, b, c\},\{b, c, d\} \in M$. Some vertex in $M$ must not contain both $b$ and $c$, since $M$ is not a triple chain. Assume that this vertex is $\{e, f, g\}$. Then $\{e, f, g\} \cap$ $\{a, b, c\}$ is either $\{a, b\}$ or $\{a, c\}$, so $a \in\{e, f, g\}$. Similarly, $\{e, f, g\} \cap\{b, c, d\}$ is either $\{b, d\}$ or $\{c, d\}$, which means that $d \in\{e, f, g\}$. The previous arguments also imply that either $b \in\{e, f, g\}$ or $c \in\{e, f, g\}$. Thus $\{e, f, g\}$ is either $\{a, b, d\}$ or $\{a, c, d\}$. Finally notice that $M$ cannot contain an element $\{b, c, h\}$, where $h \notin\{a, d\}$, since it would violate Corollary 12 . Therefore $M$ contains either three or four elements, and $|S|=4$.

THEOREM 16. If $G$ is a diameter two graph and there exists an unsolvable configuration $C$ such that $\left|V_{2}\right|=k$, then $n \geq\left(\begin{array}{c}k+2 \\ 2\end{array}\right)-\lfloor k / 2\rfloor$.

Proof. Let $l$ be the number of triple chains and $a_{i}$ be the number of vertices in triple chain $i$ for $i \in\{1, \ldots, l\}, m$ be the number of minals, and $p$ be the number of pairs of elements of $V_{2}$ that are neighbors of each other but that are not adjacent to any vertex in $T$.

Since $G$ is unsolvable there is an unreachable vertex $r$. Lemma 14 implies that for every vertex in $V_{2} \cup T$ there is another unique vertex in $G$ to guarantee it has diameter two. These facts imply $n \geq 1+2\left|V_{2} \cup T\right|+|P|$. Every pair of vertices in $V_{2}$ must either be neighbors or be adjacent to the same vertex in $P$ or $T$. Each minal covers exactly six pairs, and each triple chain of length $a_{i}$ covers exactly $2 a_{i}+1$ pairs, so $|P| \geq\left(\begin{array}{c}k \\ 2\end{array}\right)-\sum_{i=1}^{l}\left(2 a_{i}+1\right)-p-6 m$. Since minals with four elements still cover only six pairs, we will assume that minals are of size 3 . Then the minimum number of vertices that must be in $G$ is

$$
\begin{aligned}
n & \geq \overbrace{1}^{r}+2(\overbrace{k}^{\left|V_{2}\right|}+\overbrace{\sum_{i=1}^{l} a_{i}+3 m}^{|T|})+\overbrace{\left(\begin{array}{l}
k \\
2
\end{array}\right)-\sum_{i=1}^{l}\left(2 a_{i}+1\right)-p-6 m}^{|P|} \\
& =1+2 k+\left(\begin{array}{c}
k \\
2
\end{array}\right)-l-p \\
& =\left(\begin{array}{c}
k+2 \\
2
\end{array}\right)-l-p .
\end{aligned}
$$

This is minimized by maximizing $l+p$. Notice that Lemma 13 implies that $k \geq 3 l+2 p$, so $l+p$ is maximized when $l=0$ and $p=\lfloor k / 2\rfloor$. Thus the number of vertices in an unsolvable diameter two graph with $k$ vertices that have two pebbles is at least $\left(\begin{array}{c}k+2 \\ 2\end{array}\right)-\lfloor k / 2\rfloor$.

This theorem and Lemma 10 prove Theorem 8.

Corollary 17. A configuration $C$ on a diameter two graph $G$ with $n$ vertices is solvable if $\left|V_{2}\right|>\sqrt{2 n-1}-1$.

4. D2-Reachable is NP-complete. In this section we will reduce a restricted form of 3-SAT to REACHABLE, reproving a result due to Milans and Clark [10], who use an intermediate reduction to a form of reachability where moving more than once between any two vertices is prohibited. Our reduction, while similar, avoids this intermediate step. This is made possible by a special property of our construction: no vertex can accumulate three or more pebbles after any sequence of pebbling moves. This simplifies our proof and allows us to prove that D2-REACHABLE is NP-complete.

4.1. The construction. The restricted form of 3-SAT is the same as that used in [10]. A Boolean expression $\Phi$ is an instance of R3-SAT if the following hold: 
1. $\Phi$ has at least two clauses.

2. Each clause of $\Phi$ contains two or three variables.

3. Each variable appears either once or twice in its positive form.

4. Each variable appears exactly once in its negative form.

The authors of [10] prove that determining the satisfiability of an expression in this form is NP-complete.

Let $\Phi$ be an instance of R3-SAT with $m$ clauses in the $n$ variables $x_{1}, \ldots, x_{n}$. We will define a function that maps $\Phi$ to an instance of Reachable. To do so, we will construct a graph $G_{\Phi}=\left(V_{\Phi}, E_{\Phi}\right)$ and a configuration $C$. As we describe our construction, refer to Figure 2 for an illustrative example.

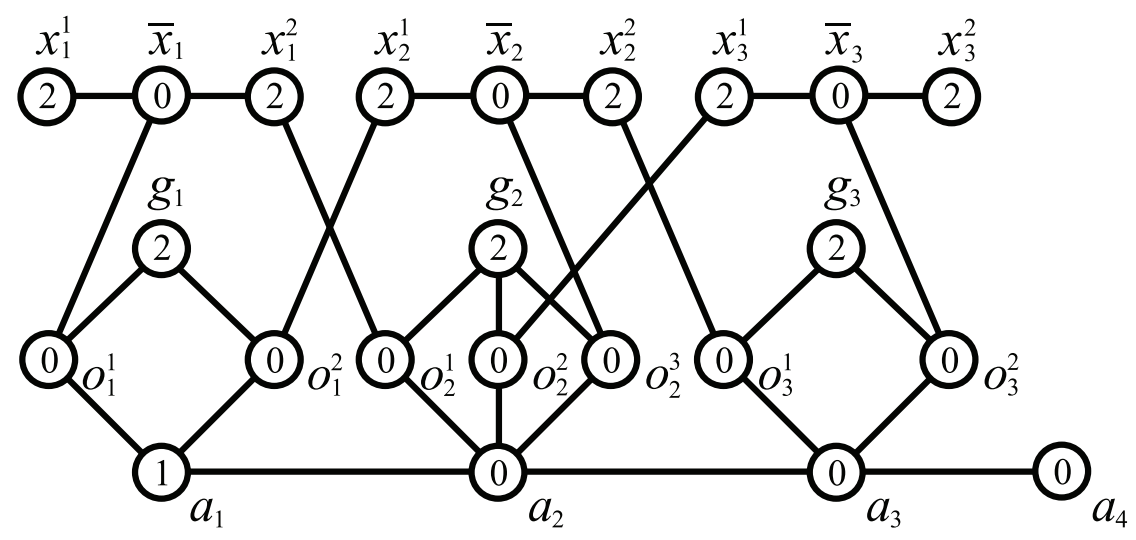

FIG. 2. $G_{\Phi}$ and $C$ for $\Phi=\left(\bar{x}_{1} \vee x_{2}\right) \wedge\left(x_{1} \vee \bar{x}_{2} \vee x_{3}\right) \wedge\left(x_{2} \vee \bar{x}_{3}\right)$, with the number of pebbles indicated within the vertex.

For each variable $x_{i}$, we will create a variable gadget: construct three vertices $x_{i}^{1}$, $\bar{x}_{i}$, and $x_{i}^{2}$, with edges $\left\{x_{i}^{1}, \bar{x}_{i}\right\}$ and $\left\{\bar{x}_{i}, x_{i}^{2}\right\}$. Vertices $x_{i}^{1}$ and $x_{i}^{2}$ correspond to positive instances of $x_{i}$, and $\bar{x}_{i}$ corresponds to the negative instance.

For each clause $\mathcal{C}_{j}$, containing literals $t_{1}, t_{2}$, and possibly $t_{3}$, construct a corresponding $O R$ gadget: create a vertex $g_{j}$ and, for each literal $t_{k}$ in $\mathcal{C}_{j}$, create a vertex $o_{j}^{k}$. We will refer to the set of such $o_{j}^{k}$ as $O_{j}$. For all $j \in\{1, \ldots, m\}$ connect $g_{j}$ to each $v \in O_{j}$, and connect each such $v$ to a corresponding vertex in a variable gadget. That is, if $t_{k}$ is $x_{i}$, connect $v$ to one of $x_{i}^{1}$ and $x_{i}^{2}$. If $t_{k}$ is $\bar{x}_{i}$, instead connect $v$ to $\bar{x}_{i}$. In doing so, connect each vertex in each variable gadget to at most one vertex in $\bigcup_{j} O_{j}$. This can be done because each variable appears once in its negative form and once or twice in its positive form. It should be noted that there is no correspondence between the subscripts and/or superscripts in the variable gadgets and OR gadgets.

Finally, for $j \in\{1, \ldots, m\}$ construct vertex $a_{j}$ and connect $a_{j}$ to each $o_{j}^{k}$. For $j \in\{2, \ldots, m\}$ connect $a_{j}$ to $a_{j-1}$. As a target vertex, construct $r=a_{m+1}$, and connect $r$ to $a_{m}$.

Define the configuration $C$ as follows: for $i \in\{1, \ldots, n\}$, let $C\left(x_{i}^{1}\right)=C\left(x_{i}^{2}\right)=2$. For $j \in\{1, \ldots, m\}$, let $C\left(g_{j}\right)=2$. Let $C\left(a_{1}\right)=1$. For all other $w \in V_{\Phi}, C(w)=0$.

4.2. Pebble accumulation bound. The following property of $G_{\Phi}$ and $C$ permits reduction beyond Reachable to D2-Reachable via $\mathcal{H}\left(G_{\Phi}\right)$.

Lemma 18. No sequence of pebbling moves in $G_{\Phi}$ from $C$ places three or more pebbles on any vertex in $V_{\Phi}$. 
Proof. Assume such a sequence exists, and let $\sigma$ be a minimal such sequence. Since $\sigma$ is minimal, it cannot perform the same move twice, as this would allow us to find a subsequence of $\sigma$ that places four pebbles on that move's source. (We will use this fact frequently, often implicitly.) One consequence of this fact is that $\sigma$ cannot put three pebbles on $r$, since $r$ 's only neighbor is $a_{m}$.

Next, note that $\sigma$ cannot pebble from $a_{j}$ to $a_{j-1}$ for $j \in\{2, \ldots, m+1\}$. Assume otherwise. Since $\sigma$ is acyclic, the result is clear for $j=m+1$. Let $j$ be the largest number in $\{2, \ldots, m\}$ for which $\sigma$ pebbles from $a_{j}$ to $a_{j-1}$. Because $\sigma$ is acyclic, it must be that $a_{j}$ receives two pebbles from inside $\mathcal{C}_{j}$ 's gadget. Even if each $v \in O_{j}$ receives one pebble from its neighbor in a variable gadget, it becomes apparent upon inspection that it is not possible to move two pebbles to $a_{j}$. This is a contradiction, so $\sigma$ can't pebble from $a_{j}$ to $a_{j-1}$ for $j \in\{2, \ldots, m\}$.

It follows that for $j \in\{2, \ldots, m\}, a_{j}$ can receive at most one pebble from outside $\mathcal{C}_{j}$ 's gadget (from $a_{j-1}$ ). Similarly, each $v \in O_{j}$ with $j \in\{1, \ldots, m\}$ can receive only one pebble from its variable gadget. Inspection of $\mathcal{C}_{j}$ 's gadget reveals that $\sigma$ cannot place three pebbles on any $v \in O_{j} \cup\left\{a_{j}, g_{j}\right\}$ for $j \in\{1, \ldots, m\}$.

Similarly, notice that for all $j \in\{1, \ldots, m\}, \sigma$ cannot pebble from $v \in O_{j}$ to a vertex $w$ in a variable gadget: even if each other vertex in $O_{j}$ receives one pebble from outside $\mathcal{C}_{j}$ 's gadget, and $a_{j}$ also receives a pebble from outside the gadget (if $j \in\{2, \ldots, m\})$, inspection once more reveals that $\sigma$ cannot place two pebbles on $v$ without pebbling from $w$ to $v$.

As a result of the previous fact, if $\sigma$ places three pebbles on a vertex in a variable gadget, it must do so using only pebbles from that gadget. The structure of the variable gadgets makes this impossible. It follows, then, that $\sigma$ cannot place three pebbles on any vertex in $V_{\Phi}$, a contradiction. It follows that no sequence of pebbling moves from $C$ places three or more pebbles on any vertex in $V_{\Phi}$.

Corollary 19. No sequence of pebbling moves in $G_{\Phi}$ from $C$ uses the same move twice.

4.3. The main results. Finally we are ready for the main results of the paper.

Theorem 20. Reachability is NP-complete.

Proof. It is clear that ReAchability is in NP. To prove that it is NP-hard, we will show that our construction is a polynomial-time reduction. Suppose that $f:\left\{x_{1}, \ldots, x_{n}\right\} \rightarrow\{$ true, false $\}$ is a satisfying truth assignment for $\Phi$. For $\Phi$ to be true, each clause in $\Phi$ must also evaluate to true, which in turn implies that one of the literals in each clause must evaluate to true.

For each variable $x_{i}$ for which $f\left(x_{i}\right)=$ false, perform pebbling moves from $x_{i}^{1}$ and $x_{i}^{2}$ to $\bar{x}_{i}$. Since $f$ is a satisfying truth assignment, we can choose one $w_{j} \in O_{j}$ for each $j \in\{1, \ldots, m\}$ that is adjacent to a vertex in a variable gadget that has two pebbles. Each $w_{j}$ can then receive one pebble from the variable gadget and one from $g_{j}$, allowing one pebble to be placed on $a_{j}$.

Notice that $a_{1}$ has two pebbles and each $a_{j}$ for $j \in\{2, \ldots, m\}$ has one pebble, so we can pebble along the path of length $m$ created by the $a_{j}$ 's to reach $a_{m+1}=r$.

Conversely, assume there exists a sequence of pebbling moves that places a pebble on $r$. Choose such a sequence $\sigma$ of minimum length. By Lemma 1, $\sigma$ cannot contain any cycles.

Note that $\sigma$ must pebble from $a_{j}$ to $a_{j+1}$ for each $j \in\{1, \ldots, m\}$. Assume otherwise. Clearly $\sigma$ must pebble from $a_{m}$ to $r=a_{m+1}$. Let $j$ be the largest number in $\{2, \ldots, m\}$ such that $\sigma$ does not pebble from $a_{j-1}$ to $a_{j}$. Since $\sigma$ pebbles from $a_{j}$ to $a_{j+1}$, and is acyclic, $a_{j}$ must receive two pebbles from a vertex in $O_{j}$. But as was 
argued in the proof of Lemma 18, this is impossible given the structure of the gadgets.

Since $\sigma$ is acyclic, Corollary 19 also implies that, for $j \in\{1, \ldots, m\}, a_{j}$ receives one pebble from a vertex in $O_{j}$. By the way these gadgets were constructed this can occur only if $\sigma$ pebbles from a variable gadget to each clause gadget. Notice that $\sigma$ cannot move a pebble from both $\bar{x}_{i}$ and either of $x_{i}^{1}$ or $x_{i}^{2}$. Construct a truth assignment $f$ as follows: if $\sigma$ pebbles from $\bar{x}_{i}$ to a vertex in a clause gadget, then $f\left(x_{i}\right)=$ false. For each other variable $x_{i}$, set $f\left(x_{i}\right)$ to true. Since each clause gadget receives a pebble from a variable gadget, the corresponding clause is satisfied, so $f$ is a satisfying truth assignment. Therefore, there exists a satisfying truth assignment for $\Phi$ if and only if it is possible to pebble to $r$ in $G_{\Phi}$ from $C$.

Note that $\left|V_{\Phi}\right| \leq 5 m+3 n+1$, and since the degree of every vertex is no more than $5,\left|E_{\Phi}\right| \leq \frac{5}{2}\left|V_{\Phi}\right|$, so the size of $G_{\Phi}$ is clearly linear in the size of $\Phi$. Also, for all $v \in V_{\Phi}, C(v) \leq 2$, so the number of pebbles is linear in the size of $G_{\Phi}$, and therefore the size of $\Phi$. Thus it is clear that our reduction can be constructed in polynomial time in the size of $\Phi$.

Theorem 21. D2-Reachable is NP-complete.

Proof. Since D2-Reachable is a special case of Reachable, which is in NP, D2-REACHABLE is also in NP.

Given an instance of R3-SAT $\Phi$, consider $\mathcal{H}\left(G_{\Phi}\right)$. Recall that, by Lemma 4, $\mathcal{H}\left(G_{\Phi}\right)$ has diameter two. We saw in the proof of Theorem 20 that $r$ is reachable in $G_{\Phi}$ from $C$ if and only if $\Phi$ was satisfiable. Lemmas 18 and 5 together imply that $r$ is reachable from $C$ in $G_{\Phi}$ if and only if $r$ is reachable from $C^{\prime}$ in $\mathcal{H}\left(G_{\Phi}\right)$. It follows that $\Phi$ is satisfiable if and only if $r$ is reachable from $C^{\prime}$ in $\mathcal{H}\left(G_{\Phi}\right)$.

All that remains to be shown is that $\mathcal{H}\left(G_{\Phi}\right)$ and $C^{\prime}$ can be constructed in polynomial time in the size of $\Phi$. Our proof of Theorem 20 included the fact that the reduction from $\Phi$ to $G_{\Phi}$ and $C$ can be constructed in polynomial time. The number of vertices in $\mathcal{H}\left(G_{\Phi}\right)$ is $O\left(\left|V_{\Phi}\right|^{2}\right)$, and the number of edges is at most the square of the number of vertices, so the size of $H\left(G_{\Phi}\right)$ is polynomial in the size of $\Phi$. Since $C^{\prime}$ has only the pebbles from $C$, it is clear that the reduction can be computed in polynomial time.

5. Conclusion. This paper provides a somewhat counterintuitive result: although Pebbling-Number goes from being $\Pi_{2}^{p}$-complete in general to being $\mathrm{P}$ for diameter two graphs, REACHABLE is NP-complete regardless of the diameter of a graph (except for diameter one, of course). Since, at least on the surface, computing pebbling number requires determining solvability, this seems rather odd. Of course determining the pebbling number really requires only determining the solvability of configurations of specific sizes, and this apparently makes a big difference in this case.

Acknowledgments. The authors thank the reviewers for several useful comments, including a suggestion that greatly simplified the proof of Lemma 3.

\section{REFERENCES}

[1] A. Bekmetjev, G. Brightwell, A. Czygrinow, and G. Hurlbert, Thresholds for families of multisets, with an application to graph pebbling, Discrete Math., 269 (2003), pp. 21-34.

[2] A. Bekmetjev and C. A. Cusack, Pebbling algorithms in diameter two graphs, SIAM J. Discrete Math., 23 (2009), pp. 634-646.

[3] A. Blasiak and J. Schmitt, Degree sum conditions in graph pebbling, Australas. J. Combin., 42 (2008), pp. 83-90.

[4] F. R. K. Chung, Pebbling in hypercubes, SIAM J. Discrete Math., 2 (1989), pp. 467-472. 
[5] T. A. Clarke, R. A. Hochberg, and G. H. Hurlbert, Pebbling in diameter two graphs and products of graphs, J. Graph Theory, 25 (1997), pp. 119-128.

[6] D. Herscovici, B. Hester, And G. Hurlbert, t-pebbling and extensions, Graphs Combin., March 2012 (electronic).

[7] G. Hurlbert, A survey of graph pebbling, Congr. Numer., 139 (1999), pp. 41-64.

[8] G. Hurlbert, Recent progress in graph pebbling, Graph Theory Notes N.Y., XLIX (2005), pp. $25-37$.

[9] G. Hurlbert and H. Kierstead, On the Complexity of Graph Pebbling, unpublished manuscript, School of Mathematical and Statistical Sciences, Arizona State University, 2005.

[10] K. Milans and B. Clark, The complexity of graph pebbling, SIAM J. Discrete Math., 20 (2006), pp. 769-798.

[11] N. G. Watson, The Complexity of Pebbling and Cover Pebbling, preprint, 2005, online at arXiv:math/0503511v3. 